\title{
SOME PROPERTIES OF CONVOLUTION FOR HYPERGEOMETRIC DISTRIBUTION TYPE SERIES ON CERTAIN ANALYTIC UNIVALENT FUNCTIONS
}

\author{
S. Porwal, A. Gupta
}

ABstract. The purpose of the present paper is to obtain certain sufficient conditions for hypergeometric distribution type series on certain analytic univalent functions.

2010 Mathematics Subject Classification: 30C45.

Keywords: Starlike, Convex functions, Hypergeometric functions and Probability Distribution.

\section{INTRODUCTION}

Let $\mathcal{A}$ denote the class of functions $f$ of the form

$$
f(z)=z+\sum_{n=2}^{\infty} a_{n} z^{n},
$$

which are analytic in the open unit $\operatorname{disc} U=\{z: z \in C$ and $|z|<1\}$ and satisfy the normalization condition $f(0)=f^{\prime}(0)-1=0$. Further, we denote by $S$ the subclass of $\mathcal{A}$ consisting of functions of the form (1) which are also univalent in $U$.

A function $f \in \mathcal{A}$ is said to be in the class $R^{\tau}(A, B)$, if

$$
\left|\frac{f^{\prime}(z)-1}{\tau(A-B)-B\left(f^{\prime}(z)-1\right)}\right|<1, \quad(-1 \leq B<A \leq 1 ; \tau \in C /\{0\} ; z \in U),
$$

clearly, a function $f$ belong to $R^{\tau}(A, B)$, if and only if there exist a function $\omega$ regular in $U$ satisfying $\omega(0)=0$ and

$$
|\omega(z)|<1, \quad(z \in U) \text { such that }
$$




$$
1+\frac{1}{\tau}\left(f^{\prime}(z)-1\right)=\frac{1+A \omega(z)}{1+B \omega(z)}, \quad(z \in U),
$$

the class $R^{\tau}(A, B)$ was introduced by Dixit and Pal [3].

A function $f$ of the form (1) is said to be starlike of order $\alpha$ if it satisfies the following condition

$$
\Re\left\{\frac{z f^{\prime}(z)}{f(z)}\right\}>\alpha, \quad z \in U,
$$

the classes of all starlike functions are denoted by $S^{*}(\alpha)$, studied by Robertson [14] and Silverman [15].

For $\lambda>0$, Ponnusamy and Rønning [10] introduced the classes $S_{\lambda}^{*}$ and $C_{\lambda}$ consisting of functions of the form (1) as follows

$$
S_{\lambda}^{*}=\left\{f \in \mathcal{A}:\left|\frac{z f^{\prime}(z)}{f(z)}-1\right|<\lambda, \quad z \in U\right\}
$$

and

$$
C_{\lambda}=\left\{f \in \mathcal{A}: z f^{\prime}(z) \in S_{\lambda}^{*}\right\} .
$$

It is obvious that $f \in C_{\lambda}$ if and only if $z f^{\prime}(z) \in S_{\lambda}^{*}$.

Recently, Porwal [11] gives a beautiful application of Poission distribution series on these subclasses. They establishes a connection between probability distribution and complex analysis and opened up a new direction of research in Geometric Function Theory. After the appearence of this paper many researchers e.g. (Murugusundaramoorthy [8], Murugusundaramoorthy et al. [9], Porwal and Kumar [12], [13] etc.) obtained certain necessary and sufficient conditions for Poisson distribution series, confluent hypergeometric distribution series, hypergeometric distribution type series etc. belonging to the various subclasses of univalent functions. Motivating with the above mentioned work, we obtain neccessary and sufficient conditions for hypergeometric distribution type series belonging to the classes $R^{\tau}(A, B), S_{\lambda}^{*}$, $C_{\lambda}, U C V, U S T$ and $k-U C V(\alpha)$.

Very recently, Ahmad [1] introduce hypergeometric type distribution as follows, for this purpose we recall the definition of hypergeometric series. The power series

$$
\sum_{n=0}^{\infty} \frac{(a)_{n}(b)_{n}}{(c)_{n}(1)_{n}} z^{n}
$$


where $a, b, c$ are complex numbers such that $c \neq 0,-1,-2, \ldots \ldots$ and $(a)_{n}$ is the Pochhammer symbol defined in terms of the Gamma function by,

$$
\begin{aligned}
(a)_{n} & =\frac{\Gamma(a+n)}{\Gamma(a)} \\
& = \begin{cases}1, & \text { if } n=0 \\
a(a+1) \ldots(a+n-1), & \text { if } n \in N=\{1,2,3, \ldots\}\end{cases}
\end{aligned}
$$

is convergent for all finite value of $z$. is called the Hypergeometric series. The series converges absolutly if $|z|<1$ and diverges if $|z|>1$ and for $|z|=1$ the series is absolutly convergent if $\Re(c-a-b)>0$. It is denoted by $F(a, b ; c ; z)$.

Now we define for $a, b, c, m>0$ such that the series,

$$
F(a, b ; c ; m)=\sum_{n=0}^{\infty} \frac{(a)_{n}(b)_{n}}{(c)_{n}(1)_{n}} m^{n}
$$

is convergent.

Now we introduce hypergeometric type distribution whose probability mass function is,

$$
\frac{(a)_{n}(b)_{n}}{(c)_{n} n ! F(a, b ; c ; m)} m^{n}
$$

Now we introduce a new series $I(a, b ; c ; m ; z)$ in the following way,

$$
I(a, b ; c ; m ; z)=z+\sum_{n=2}^{\infty} \frac{(a)_{n-1}(b)_{n-1} m^{n-1}}{(c)_{n-1}(n-1) ! F(a, b ; c ; m)} z^{n},
$$

where $a, b, c, m>0$. The series $I(a, b ; c ; m ; z)$ is absolutly convergent for $|z|<1$.

The convolution (or Hadamard product) of two series

$$
f(z)=\sum_{n=0}^{\infty} a_{n} z^{n}
$$

and

$$
g(z)=\sum_{n=0}^{\infty} b_{n} z^{n}
$$

is defined as the power series,

$$
(f * g)(z)=\sum_{n=0}^{\infty} a_{n} b_{n} z^{n}
$$


S. Porwal, A. Gupta - Some properties of convolution for hypergeometric ...

Now we consider a linear operator,

$$
K(a, b ; c ; m ; z) f(z)=z+\sum_{n=2}^{\infty} \frac{(a)_{n-1}(b)_{n-1} m^{n-1}}{(c)_{n-1}(n-1) ! F(a, b ; c ; m)} a_{n} z^{n} .
$$

Bharti et al. [2] introduced the subclass of k-uniformly convex functions of order $\alpha$ and corressponding class of starlike functions as follows-

If $f \in \mathcal{A}, 0 \leq k<\infty$ and $0 \leq \alpha<1$ then $f \in k-U C V(\alpha)$, if and only if

$$
\Re\left\{1+\frac{z f^{\prime \prime}(z)}{f^{\prime}(z)}\right\} \geq k\left|\frac{z f^{\prime \prime}(z)}{f^{\prime}(z)}\right|+\alpha .
$$

For $\alpha=0$ the class $k-U C V(\alpha)$ reduce to the class $k-U C V$ introduced and studied by Kanas and Wisniowska [6] and for $k=1, \alpha=0$ it reduce to the class uniformly convex function $U C V$ studied by Goodman [5], (see also [4], [7] and [16]).

To prove our main results we shall require the following lemmas.

Lemma 1. ([3]) Let a function $f$ of the form (1) be in $R^{\tau}(A, B)$. Then,

$$
\left|a_{n}\right| \leq \frac{(A-B)|\tau|}{n} .
$$

The result is sharp for the function

$$
f(z)=\int_{0}^{z}\left(1+\frac{(A-B) \tau z^{n-1}}{1+B z^{n-1}}\right) d z, \quad(n \geq 2 ; z \in U) .
$$

Lemma 2. ([3]) Let a function $f$ of the form (1) be in $\mathcal{A}$. If

$$
\sum_{n=2}^{\infty}(1+|B|) n\left|a_{n}\right| \leq(A-B)|\tau|, \quad(-1 \leq B<A \leq 1 ; \tau \in C) .
$$

Then $f \in R^{\tau}(A, B)$.

The result is sharp for the function

$$
f(z)=z+\frac{(A-B) \tau}{(1+|B|) n} z^{n}, \quad(n \geq 2 ; z \in U) .
$$

Lemma 3. ([10]) Let $f \in A$ be of the form (1). If

$$
\sum_{n=2}^{\infty}(\lambda+n-1)\left|a_{n}\right| \leq \lambda, \quad(\lambda>0)
$$

then $f \in S_{\lambda}^{*}$. 
Lemma 4. [10] Let $f \in A$ be of the form (1). If

$$
\sum_{n=2}^{\infty} n(\lambda+n-1)\left|a_{n}\right| \leq \lambda, \quad(\lambda>0),
$$

then $f \in C_{\lambda}$.

Lemma 5. ([5]) A function $f$ of the form (1) is in $U C V$ if

$$
\sum_{n=2}^{\infty} n(2 n-1)\left|a_{n}\right| \leq 1 \text {. }
$$

Lemma 6. ([5]) A function $f$ of the form (1) is in UST if

$$
\sum_{n=2}^{\infty}(3 n-2)\left|a_{n}\right| \leq 1 .
$$

Lemma 7. ([2]) A function $f \in A$ is in $k-U C V(\alpha)$ if it satisfies the following condition

$$
\sum_{n=2}^{\infty} n[n(1+k)-(k+\alpha)]\left|a_{n}\right| \leq 1-\alpha .
$$

\section{Main Results}

Theorem 8. Let $a, b, c>0$ and $c>a+b, m \in(0,1)$. Suppose that $f \in R^{\tau}(A, B)$ and satisfy the condition

$$
F(a, b ; c ; m) \leq 1+\frac{1}{|B|},
$$

then the operator $K(a, b ; c ; m ; z)$ maps $R^{\tau}(A, B)$ into $R^{\tau}(A, B)$.

Proof. Let $a, b, c>0$ and $m \in(0,1)$.

Suppose that $f(z)=z+\sum_{n=2}^{\infty} a_{n} z^{n} \in R^{\tau}(A, B)$.

Then by Lemma 2, it sufficeint to show that,

$$
T_{1}=\sum_{n=2}^{\infty}(1+|B|) n\left|A_{n}\right| \leq(A-B)|\tau|,
$$


S. Porwal, A. Gupta - Some properties of convolution for hypergeometric ...

where

$$
\begin{gathered}
A_{n}=\frac{(a)_{n-1}(b)_{n-1}}{(c)_{n-1}(n-1) !} \frac{m^{n-1}}{F(a, b ; c ; m)} a_{n} . \\
T_{1}=\sum_{n=2}^{\infty}(1+|B|) n \frac{(a)_{n-1}(b)_{n-1}}{(c)_{n-1}(n-1) !} \frac{m^{n-1}}{F(a, b ; c ; m)}\left|a_{n}\right| \\
\leq(1+|B|) \sum_{n=2}^{\infty} \frac{n(a)_{n-1}(b)_{n-1}}{(c)_{n-1}(n-1) !} \frac{m^{n-1}}{F(a, b ; c ; m)} \frac{(A-B)|\tau|}{n} \\
=\frac{(A-B)|\tau|(1+|B|)}{F(a, b ; c ; m)} \sum_{n=2}^{\infty} \frac{(a)_{n-1}(b)_{n-1}}{(c)_{n-1}(n-1) !} m^{n-1} \\
=\frac{(A-B)|\tau|(1+|B|)}{F(a, b ; c ; m)}(F(a, b ; c ; m)-1) \\
\leq(A-B)|\tau|,
\end{gathered}
$$

by the given hypothesis.

Thus the operator $K(a, b ; c ; m ; z)$ maps $R^{\tau}(A, B)$ into $R^{\tau}(A, B)$.

Theorem 9. Let $a, b, c>1$ and $c>a+b, m \in(0,1)$. Suppose that $f \in R^{\tau}(A, B)$ and satisfy the condition

$$
\begin{aligned}
& \frac{|A-B \| \tau|}{F(a, b ; c ; m)}\{F(a, b ; c ; m)-1\}+\frac{(\lambda-1)(c-1)}{(a-1)(b-1) m}\left\{F(a-1, b-1 ; c-1 ; m)-1-\frac{(a-1)(b-1)}{(c-1)} m\right\} \\
& \leq \lambda,
\end{aligned}
$$

then the operator $K(a, b ; c ; m ; z)$ maps $R^{\tau}(A, B)$ into $S_{\lambda}^{*}$.

Proof. Let $a, b, c>1$ and $m \in(0,1)$. Suppose that $f(z)=z+\sum_{n=2}^{\infty} a_{n} z^{n} \in R^{\tau}(A, B)$. Then by Lemma 3 it is sufficient to show that

$$
\begin{gathered}
T_{2}=\sum_{n=2}^{\infty}(n+\lambda-1)\left|A_{n}\right| \leq \lambda \\
=\sum_{n=2}^{\infty}(n+\lambda-1) \frac{(a)_{n-1}(b)_{n-1}}{(c)_{n-1}(n-1) !} \frac{m^{n-1}}{F(a, b ; c ; m)}\left|A_{n}\right| \\
=\sum_{n=2}^{\infty}(n+\lambda-1)\left|\frac{(a)_{n-1}(b)_{n-1}}{(c)_{n-1}(n-1) !} m^{n-1}\right| \frac{|A-B||\tau|}{n F(a, b ; c ; m)} \\
=\frac{(A-B)|\tau|}{F(a, b ; c ; m)}\left\{\sum_{n=2}^{\infty} \frac{(a)_{n-1}(b)_{n-1}}{(c)_{n-1}(n-1) !} m^{n-1}+(\lambda-1) \sum_{n=2}^{\infty} \frac{(a)_{n-1}(b)_{n-1}}{(c)_{n-1}(n) !} m^{n-1}\right\}
\end{gathered}
$$




$$
\begin{gathered}
=\frac{|A-B \| \tau|}{F(a, b ; c ; m)}\left\{\sum_{n=1}^{\infty} \frac{(a)_{n}(b)_{n}}{(c)_{n}(n) !} m^{n}+(\lambda-1) \sum_{n=2}^{\infty} \frac{(a)_{n-1}(b)_{n-1}}{(c)_{n-1}(n) !} m^{n-1}\right\} \\
=\frac{|A-B \| \tau|}{F(a, b ; c ; m)}\left\{\sum_{n=1}^{\infty} \frac{(a)_{n}(b)_{n}}{(c)_{n}(n) !} m^{n}+\frac{(\lambda-1)(c-1)}{(a-1)(b-1) m} \sum_{n=2}^{\infty} \frac{(a-1)_{n}(b-1)_{n}}{(c-1)_{n}(n) !} m^{n}\right\} \\
=\frac{|A-B \| \tau|}{F(a, b ; c ; m)}\{F(a, b ; c ; m)-1\}+\frac{(\lambda-1)(c-1)}{(a-1)(b-1) m} \\
\left\{F(a-1, b-1 ; c-1 ; m)-1-\frac{(a-1)(b-1)}{(c-1)} m\right\} \\
\leq \lambda,
\end{gathered}
$$

by the given hypothesis.

Thus the operator $K(a, b ; c ; m ; z)$ maps $R^{\tau}(A, B)$ into $S_{\lambda}^{*}$.

Theorem 10. Let $a, b, c>0$ and $c>a+b, m \in(0,1)$. Suppose that $f \in R^{\tau}(A, B)$ and satisfy the condition

$$
(A-B)|\tau|\left\{\frac{a b}{c} m F(a+1, b+1 ; c+1 ; m)+\lambda\{F(a, b ; c ; m)-1\}\right\} \leq \lambda F(a, b ; c ; m),
$$

then the operator $K(a, b ; c ; m ; z)$ maps $R^{\tau}(A, B)$ into $C_{\lambda}$.

Proof. Let $a, b, c>0$ and $m \in(0,1)$. Suppose that $f(z)=z+\sum_{n=2}^{\infty} a_{n} z^{n} \in R^{\tau}(A, B)$. Then by Lemma 4 . It is sufficient to show that

$$
T_{3}=\sum_{n=2}^{\infty} n(n+\lambda-1)\left|A_{n}\right| \leq \lambda .
$$

Now

$$
\begin{gathered}
T_{3}=\sum_{n=2}^{\infty} n(n+\lambda-1)\left|\frac{(a)_{n-1}(b)_{n-1}}{(c)_{n-1}(n-1) !} m^{n-1} \frac{a_{n}}{F(a, b ; c ; m)}\right| \\
\leq \sum_{n=2}^{\infty} n(n+\lambda-1) \frac{(a)_{n-1}(b)_{n-1}}{(c)_{n-1}(n-1) !} \frac{m^{n-1}}{F(a, b ; c ; m)} \frac{(A-B)|\tau|}{n} \\
=\frac{(A-B)|\tau|}{F(a, b ; c ; m)}\left\{\sum_{n=2}^{\infty}\{(n-1)+\lambda\} \frac{(a)_{n-1}(b)_{n-1}}{(c)_{n-1}(n-1) !} m^{(n-1)}\right\} \\
=\frac{(A-B)|\tau|}{F(a, b ; c ; m)}\left\{\sum_{n=2}^{\infty}(n-1) \frac{(a)_{n-1}(b)_{n-1}}{(c)_{n-1}(n-1) !} m^{n-1}+\lambda \sum_{n=2}^{\infty} \frac{(a)_{n-1}(b)_{n-1}}{(c)_{n-1}(n-1) !} m^{n-1}\right\}
\end{gathered}
$$




$$
\begin{gathered}
=\frac{(A-B)|\tau|}{F(a, b ; c ; m)}\left\{\sum_{n=2}^{\infty} \frac{(a)_{n-1}(b)_{n-1}}{(c)_{n-1}(n-2) !} m^{n-1}+\lambda \sum_{n=1}^{\infty} \frac{(a)_{n}(b)_{n}}{(c)_{n}(n) !} m^{n}\right\} \\
=\frac{(A-B)|\tau|}{F(a, b ; c ; m)}\left\{\frac{a b}{c} m \sum_{n=2}^{\infty} \frac{(a+1)_{n-2}(b+1)_{n-2}}{(c+1)_{n-2}(n-2) !} m^{n-2}+\lambda\{F(a, b ; c ; m)-1\}\right\} \\
=\frac{(A-B)|\tau|}{F(a, b ; c ; m)}\left\{\frac{a b}{c} m F(a+1, b+1 ; c+1 ; m)+\lambda\{F(a, b ; c ; m)-1\}\right\} \\
\leq \lambda,
\end{gathered}
$$

by the given hypothesis.

Thus the operator $K(a, b ; c ; m ; z)$ maps $R^{\tau}(A, B)$ into $C_{\lambda}$.

Theorem 11. Let $a, b, c>0$ and $c>a+b, m \in(0,1)$. Suppose that $f \in R^{\tau}(A, B)$ and satisfy the condition

$$
(A-B)|\tau|\left\{2 \frac{a b}{c} m F(a+1, b+1 ; c+1 ; m)+F(a, b ; c ; m)-1\right\} \leq F(a, b ; c ; m),
$$

then the operator $K(a, b ; c ; m ; z)$ maps $R^{\tau}(A, B)$ into $U C V$.

Proof. Let $a, b, c>0$ and $m \in(0,1)$. Suppose that $f(z)=z+\sum_{n=2}^{\infty} a_{n} z^{n} \in R^{\tau}(A, B)$. Then by Lemma 5 , we have

$$
\begin{gathered}
T_{4}=\sum_{n=2}^{\infty} n(2 n-1) \frac{(a)_{n-1}(b)_{n-1}}{(c)_{n-1}(n-1) !} \frac{m^{n-1}}{F(a, b ; c ; m)}\left|a_{n}\right| \\
\leq \sum_{n=2}^{\infty} n(2 n-1) \frac{(a)_{n-1}(b)_{n-1}}{(c)_{n-1}(n-1) !} m^{n-1} \frac{(A-B)|\tau|}{n F(a, b ; c ; m)} \\
=\frac{(A-B)|\tau|}{F(a, b ; c ; m)} \sum_{n=2}^{\infty}\{2(n-1)+1\} \frac{(a)_{n-1}(b)_{n-1}}{(c)_{n-1}(n-1) !} m^{n-1} \\
=\frac{(A-B)|\tau|}{F(a, b ; c ; m)}\left\{2 \sum_{n=2}^{\infty}(n-1) \frac{(a)_{n-1}(b)_{n-1}}{(c)_{n-1}(n-1) !} m^{n-1}+\sum_{n=2}^{\infty} \frac{(a)_{n-1}(b)_{n-1}}{(c)_{n-1}(n-1) !} m^{n-1}\right\} \\
=\frac{(A-B)|\tau|}{F(a, b ; c ; m)}\left\{2 \sum_{n=2}^{\infty} \frac{(a)_{n-1}(b)_{n-1}}{(c)_{n-1}(n-2)} m^{n-1}+\sum_{n=1}^{\infty} \frac{(a)_{n}(b)_{n}}{(c)_{n}(n) !} m^{n}\right\} \\
=\frac{(A-B)|\tau|}{F(a, b ; c ; m)}\left\{2 \frac{a b}{c} m \sum_{n=0}^{\infty} \frac{(a+1)_{n}(b+1)_{n}}{(c+1)_{n}(n) !} m^{n}+F(a, b ; c ; m)-1\right\}
\end{gathered}
$$


S. Porwal, A. Gupta - Some properties of convolution for hypergeometric ...

$$
\begin{gathered}
=\frac{(A-B)|\tau|}{F(a, b ; c ; m)}\left\{2 \frac{a b}{c} m F(a+1, b+1 ; c+1 ; m)+F(a, b ; c ; m)-1\right\} \\
\leq 1,
\end{gathered}
$$

by the given hypothesis.

Thus the operator $K(a, b ; c ; m ; z)$ maps $R^{\tau}(A, B)$ into $U C V$.

Theorem 12. Let $a, b, c>1$ and $c>a+b, m \in(0,1)$. Suppose that $f \in R^{\tau}(A, B)$ and satisfy the condition,

$$
\begin{gathered}
(A-B)|\tau|\left\{3 F(a, b ; c ; m)-1+\frac{(c-1) m}{(a-1)(b-1)} 2 F(a-1, b-1 ; c-1 ; m)-1-\frac{(a-1)(b-1)}{(c-1)}\right\} \\
\leq F(a, b ; c ; m),
\end{gathered}
$$

then the operator $K(a, b ; c ; m ; z)$ maps $R^{\tau}(A, B)$ into UST.

Proof. Let $a, b, c>0$ and $m \in(0,1)$.

Suppose that $f(z)=z+\sum_{n=2}^{\infty} a_{n} z^{n} \in R^{\tau}(A, B)$.

Then by Lemma 6 , it is sufficient to show that

$$
\begin{aligned}
& T_{5}=\sum_{n=2}^{\infty}(3 n-2)\left|\frac{(a)_{n-1}(b)_{n-1}}{(c)_{n-1}(n-1) !} \frac{m^{n-1}}{F(a, b ; c ; m)}\right| a_{n}|| \\
& =\sum_{n=2}^{\infty}(3 n-2) \frac{(a)_{n-1}(b)_{n-1}}{(c)_{n-1}(n-1) !} \frac{m^{n-1}}{F(a, b ; c ; m)}\left|a_{n}\right| \\
& \leq \sum_{n=2}^{\infty}(3 n-2) \frac{(a)_{n-1}(b)_{n-1}}{(c)_{n-1}(n-1) !} \frac{m^{n-1}}{F(a, b ; c ; m)} \frac{|A-B||\tau|}{n} \\
& =\frac{|A-B||\tau|}{F(a, b ; c ; m)}\left\{\sum_{n=2}^{\infty} 3 n \frac{(a)_{n-1}(b)_{n-1}}{(c)_{n-1}(n) !} m^{n-1}-2 \sum_{n=2}^{\infty} \frac{(a)_{n-1}(b)_{n-1}}{(c)_{n-1}(n) !} m^{n-1}\right\} \\
& =\frac{|A-B||\tau|}{F(a, b ; c ; m)}\left\{\sum_{n=2}^{\infty} 3 \frac{(a)_{n-1}(b)_{n-1}}{(c)_{n-1}(n-1) !} m^{n-1}-2 \sum_{n=2}^{\infty} \frac{(a)_{n-1}(b)_{n-1}}{(c)_{n-1} n !} m^{n-1}\right\} \\
& =\frac{(A-B)|\tau|}{F(a, b ; c ; m)}\left\{3 \sum_{n=1}^{\infty} \frac{(a)_{n}(b)_{n}}{(c)_{n} n !} m^{n}-2 \frac{(c-1)}{(a-1)(b-1)} m \sum_{n=2}^{\infty} \frac{(a-1)_{n}(b-1)_{n}}{\left.(c-1)_{(} n\right) n !} m^{n}\right\} \\
& =\frac{(A-B)|\tau|}{F(a, b ; c ; m)}\left\{3(F(a, b ; c ; m)-1)+\frac{(c-1)}{(a-1)(b-1) m} 2\right. \\
& \left.\left(F(a-1, b-1 ; c-1 ; m)-1-\frac{(a-1)(b-1)}{(c-1)} m\right)\right\}
\end{aligned}
$$




$$
\leq 1
$$

by the given hypothesis.

Thus the operator $K(a, b ; c ; m ; z)$ maps $R^{\tau}(A, B)$ into $U S T$.

Theorem 13. Let $a, b, c>0$ and $c>a+b, m \in(0,1)$. Suppose a function $f \in R^{\tau}(A, B)$ is in $k-U C V(\alpha)$ if it satisfies the following condition

$$
\frac{(A-B)|\tau|}{F(a, b ; c ; m)}\left[(k+1) \frac{a b}{c} m F(a+1, b+1 ; c+1 ; m)+(1-\alpha)[F(a, b ; c ; m)-1]\right] \leq(1-\alpha) .
$$

Then the operator $K(a, b ; c ; m ; z)$ maps $k-U C V(\alpha)$.

Proof. Let $a, b, c>0$ and $c>a+b$. Suppose a function $f \in R^{\tau}(A, B)$ is in $k-$ $U C V(\alpha)$, then by Lemma 7 , it is sufficient to show that

$$
T_{6}=\sum_{n=2}^{\infty} n[n(1+k)-(k+\alpha)]\left|A_{n}\right| \leq 1-\alpha
$$

Now

$$
\begin{gathered}
T_{6}=\sum_{n=2}^{\infty} n[n(1+k)-(k+\alpha)] \frac{(a)_{n-1}(b)_{n-1}}{(c)_{n-1}(n-1) !} \frac{m^{n-1}}{F(a, b ; c ; m)}\left(\left|a_{n}\right|\right) \\
\leq \frac{(A-B)|\tau|}{F(a, b ; c ; m)} \sum_{n=2}^{\infty} n[n(k+1)-(k+\alpha)] \frac{(a)_{n-1}(b)_{n-1}}{(c)_{n-1}(n-1) !} \frac{m^{n-1}}{n} \\
\left.=\frac{(A-B)|\tau|}{F(a, b ; c ; m)} \sum_{n=2}^{\infty}[(k+1)(n-1)+(1-\alpha)] \frac{(a)_{n-1}(b)_{n-1}}{(c)_{n-1}(n-1) !} m^{n-1}\right] \\
=\frac{(A-B)|\tau|}{F(a, b ; c ; m)}\left[(k+1) \sum_{n=2}^{\infty} \frac{(a)_{n-1}(b)_{n-1}}{(c)_{n-1}(n-2) !} m^{n-1}+(1-\alpha) \sum_{n=2}^{\infty} \frac{(a)_{n-1}(b)_{n-1}}{(c)_{n-1}(n-1) !} m^{n-1}\right] \\
=\frac{(A-B)|\tau|}{F(a, b ; c ; m)}\left[(k+1) \frac{a b}{c} m F(a+1, b+1 ; c+1 ; m)+(1-\alpha)[F(a, b ; c ; m)-1]\right] \\
\leq(1-\alpha),
\end{gathered}
$$

by the given hypothesis.

Thus the operator $K(a, b ; c ; m ; z)$ maps $k-U C V(\alpha)$. 
S. Porwal, A. Gupta - Some properties of convolution for hypergeometric ...

\section{REFERENCES}

[1] Dilshad Ahmad,Mapping properties of some convolution operator associated with some special function, M.Phil. Dissertation, C.S.J.M. University, Kanpur, India, 2014.

[2] R. Bharti, R. Parvatham and A. Swaminathan, On subclass of uniformly convex functions and corressponding class of starlike functions, Tamkang J. Math., 28(1997), $17-32$.

[3] K.K. Dixit and S.K. Pal,On a class of univalent functions related to complex order, Indian J. Pure Appl. Math., 26 (1995) 889-896.

[4] K.K. Dixit and Saurabh Porwal, On a certain class of $k$-uniformly convex functions with negative coefficients, Bull. Cal. Math. Soc., 100 (6) (2008), 639-652.

[5] A.W. Goodman, On Uniformly convex functions, Ann. Polon. Math., 56(1991), $87-92$.

[6] S.Kanas and A. Wishiwoska, Conic regions and $k$ - uniform convexity, J. Compute. Appl. Math. Pure Appl., 105(1999), 327-336.

[7] S.Kanas and A. Wishiowska, Conic region and k-starlike functions, Rev. Roum. Math, 45(2000), 647-657.

[8] G. Murugusundaramoorthy,Subclasses of starlike and convex functions involving Poisson distribution series, Afr. Mat. (2017).

[9] G. Murugusundaramoorthy, K. Vijaya and S. Porwal,Some inclusion results of certain subclasses of analytic functions associated with Poisson distribution series, Hacettepe J. Math. Stat., 45(4)(2016), 1101-1107.

[10] S. Ponnusamy and F. Rønning, Starlikeness properties for convolution involving hypergeometric series, Ann. Univ. Mariae Curie-Sklodowska, L.II, 1(16), (1998), 141155 .

[11] Saurabh Porwal, An application of a Poission Distribushan series on certain analytic functions, J. Complex Anal., Vol(2014), Art.ID984135, 1-3.

[12] Saurabh Porwal and Manish Kumar, A unified study on starlike and convex functions associated with Poisson distribution series, Afrika Matematika, 27(5-6) (2016), 1021-1027.

[13] Saurabh Porwal and Shivam Kumar, Confluent hypergeometric distribution and its applications on certain classes of univalent functions, Afrika Matematika, 28 (2017), 1-8.

[14] M.S. Robertson, On the theory of Univalent functions, Ann.Math., 37(1936), 374-408.

[15] H. Silverman, Univalent functions with negative coefficients, Proc. Amer. Math. Soc., 51(1975), 109-116. 
S. Porwal, A. Gupta - Some properties of convolution for hypergeometric ...

[16] K.G. Subramanian, G. Murugsundramoorthy, P. Balasubramaniyam and H. Silverman, Subclass of uniformly convex and uniformly starlike functions, Math, Japon., 42(1995), 512-522.

Saurabh Porwal

Lecturer Mathematics

Sri Radhey Lal Arya Inter College, Ehan, Hathras

(U.P.) India

email:saurabhjcb@rediffmail.com

Ankita Gupta

Department of Mathematics

University Institute of Engineering and Technology,

Chhatrapati Shahu Ji Maharaj University, Kanpur-208024

(U.P.), India

email: ankita.ank@gmail.com 